\title{
A NOTE ON THE LIMIT OF ORBITS ${ }^{1}$
}

\section{G. E. SCHWEIGERT}

Theorem A, stated below, is established in a recent paper ${ }^{2}$ by $\mathrm{D}$. W. Hall and the author. Various factors favoring a change in the point of view have enabled the writer to see a simpler proposition which contains Theorem A as a corollary. The purpose of this note is to prove this newer theorem and to point to its implications in the particular case shown by the example at the end.

A single valued continuous transformation $T(M)=M$ of a compact metric space onto itself is said to be pointwise periodic provided that for each point $x$ in $M$ there exists a positive integer $n$ such that $T^{n}(x)=x$. Such a transformation must be one-to-one, hence a homeomorphism. The least positive integer $n$ such that $T^{n}(x)=x$ is said to be the period of a point $x$ in $M$ and is denoted by $p(T, x)$. By the orbit of a point under $T$, or more briefly by a point-orbit, we shall mean the finite set consisting of a point $x$ of $M$ and all the images $T(x), T^{2}(x) \equiv T T(x)$, and so on, under $T$. Similarly if $X$ is a component of an invariant set $L$, that is, a set $L$ for which $T(L)=L$, then the finite sum of disjoint components $X+T(X)+T^{2}(X)+\cdots+T^{k}(X)$ is said to be the component-orbit of $X$ relative to $L$ under $T$. This extention of the notion of a point-orbit ${ }^{3}$ is possible since the property of being a component (maximal connected set) in $L$ is invariant under $T$. In all that follows the brief term component-orbit will be used instead of the original precise form in which an invariant set is chosen first. Furthermore if $G$ is such a component-orbit sets $X$ or $K$ mentioned in close proximity to $G$ may be understood without confusion to be components such as $X$ in the definition above. In other words it is best to think of each $G$ as a function of $X$ or $K$ as if it were written $G(X)$ or $G(K)$, so that these sets may be used freely without accounting for their presence each time. The lower case letters such as $x$ and $p$ will denote points, and point-orbits will bear a subscript, for example, $G_{x}$.

These two types of orbit are obviously related in such a fashion that: (a) The number of components in a $\mathrm{n}$ orbit $G$ equals at most

\footnotetext{
1 Presented in part to the Society, April 15, 1939.

2 Properties of invariant sets under pointwise periodic homeomorphisms, Duke Mathematical Journal, vol. 4 (1938), pp. 719-724.

${ }^{3}$ See L. Whyburn, Rotation groups about a set of fixed points, Fundamenta Mathematicae, vol. 28 (1937), p. 124. This is perhaps the first and certainly a most consistent and fruitful use of this extended notion.
} 
the minimum value of $p(T, x)$ for $x \in X$; (b) If $G$ has $k$ components $T^{i}(X)$ and if $G_{x}$ is a point-orbit associated with $x \in X$, then the number of points in $G_{x} \cdot T^{i}(X)$ is the same for all values of $i$ in the range 0 to $k-1$. Furthermore the set $G_{x} \cdot T^{i}(X)$ is a point-orbit under $T^{k}$ for each fixed $i$. It is to be understood that $T^{0}(X) \equiv X$ as in case for $i=0$ above.

TheOREM A. If $M$ is a compact metric space, $T(M)=M$ a pointwise periodic transformation, $\left\{G_{x_{k}}\right\}$ a convergent sequence of pointorbits under $T$ with limit set $L$, and if there is in $L$ a connected set $B$ such that $T(B)=B$, then $L$ is connected.

The following lemma is stated without proof since it is practically the same as Theorem $\mathrm{A}$ and follows from the exact argument used in the proof as published.

LEMMA 1. If $M$ is a compact metric space, $T(M)=M$ a pointwise periodic transformation, $\left\{G_{x_{k}}\right\}$ a convergent sequence of point-orbits under $T$ with limit set $L$, and if $G_{x}$ denotes any point-orbit in $L$, then $L$ has the property that for every separation ${ }^{4} L=L^{1}+L^{2}$ the sets $G_{x} \cdot L^{1}$ and $G_{x} \cdot L^{2}$ are non-vacuous.

We are now in a position to prove an extended form of this result.

LeMmA 2. If $M$ is a compact metric space, $T(M)=M$ a pointwise periodic transformation, $\left\{G_{x_{k}}\right\}$ a convergent sequence of point-orbits under $T$ with limit set $L$, and if $X$ is a component of $L$, then $L=G$ where $G$ is the component-orbit of $X$ under $T$.

Proof. Case (i): There exists an isolated component $K$ in $L$, that is, $K$ is a component which is both open and closed in $L$.

Let $G$ denote the component-orbit of $K$ and consider the equations

$$
L=G+(L-G), \quad G=K+T(K)+\cdots+T^{m}(K),
$$

$m$ a positive integer.

Now $T$ is a homeomorphism on $L$, and $K$ being isolated in $L$ implies that $T(K)$ and $T^{2}(K)$, and so on, are also isolated in $L$ so that $G$ is both open and closed in $L$. This shows that the set $L-G$ must be vacuous, for otherwise $L=G+(L-G)$ is a separation of $L$ contrary to Lemma 1. If $L-G$ is vacuous then $L=G$ and Lemma 2 is true.

Case (ii): L contains no isolated components.

We will show that this case is impossible in view of Lemma 1. Let

${ }^{4}$ We say that $M=R+S$ is a separation of $M$ provided neither $R$ nor $S$ is vacuous and $R \bar{S}=0=\bar{R} S$, where $\bar{N}$ is the closure of a set $N$. 
$X$ be any component of $L$ and let $m$ be a positive integer chosen so that $X, T(X), \cdots, T^{m}(X)$ represent the $(m+1)$ distinct components of the component-orbit $G$. Now $L$ is not connected, for otherwise $L$ is itself an isolated component in $L$, and we may therefore choose a separation of $L$; say $L=L(1)+L(2)$. Neither $L(1)$ nor $L(2)$ is an isolated component in $L$ and hence we may choose separations $L(1)=L(1,1)+L(1,2)$ and $L(2)=L(2,1)+L(2,2)$ so that $L=L(1,1)$ $+L(1,2)+L(2,1)+L(2,2)$. Each of the summands $L\left(i_{1}, i_{2}\right)$ when $i_{1}=1,2$ and $i_{2}=1,2$ is an isolated set in $L$, hence fails to be connected, and the process continues. In general

$$
L=\sum_{i_{1}, i_{2}, \cdots, i_{j}} L\left(i_{1}, i_{2}, \cdots, i_{j}\right), \quad \text { where } i_{k}=1,2,
$$

while $k$ has the range $1,2, \cdots, j$; thus $L$ is expressed as the sum of $2^{j}$ disjoint sets each isolated in $L$. Let a fixed value of $j=j^{*}$ be chosen so that $2^{j^{*}}$ is greater than $m+1$. Obviously each component of $G$ must intersect some set $L\left(i_{1}, i_{2}, \cdots, i_{j}\right)$ and must be contained in that set. Since there are more sets in $L$ than there are components in $G$ there exists a set $L^{*} \equiv L\left(I_{1}, I_{2}, \cdots, I_{\jmath}\right)$ such that $L^{*} G=0$. However $L=L^{*}+\left(L-L^{*}\right)$ is a separation of $L$ and no point-orbit in $G$ intersects $L^{*}$. This is impossible because of Lemma 1. Thus Case (ii) never holds and Lemma 2 is true.

The next and final lemma will be recognized as one having the same conclusion as Lemma 1 established however from fewer assumptions.

LEMMA $1^{*}$. If $M$ is a compact metric space, $T(M)=M$ a pointwise periodic transformation, $\left\{G_{i}\right\}$ a convergent sequence of closed component-orbits under $T$ with limit set $L$, and if $G_{x}$ denotes any point-orbit in $L$, then $L$ has the property that for every separation

$$
L=L^{1}+L^{2}
$$

the sets $G_{x} \cdot L^{1}$ and $G_{x} \cdot L^{2}$ are non-vacuous.

Proof. Assume contrary to the lemma that $L=L^{1}+L^{2}$ is a separation of $L$ and there exists a point $x$ in $L$ such that the point-orbit $G_{x}$ is contained in one side, say $L^{1}$, of the separation. In these circumstances it is possible to choose a subsequence $\left\{G_{i_{k}}\right\}$ (of the given sequence of component-orbits) which in turn allows us to choose points $x_{k} \in G_{i_{k}}$ such that the point-orbits $\left\{G_{x_{k}}\right\}$ converge to a limit set, say $L^{*}$, and the points $\left\{x_{k}\right\}$ converge to the point $x$, which defines $G_{x}$. Since the point-orbits converge, we may use Lemma 2 which informs us that the limit set $L^{*}$ is a component-orbit. Moreover each of the finite number of components of $L^{*}$ may be taken as one side of a 
separation of $L^{*}$ and this means, in view of Lemma 1 (or Lemma 2) and the fact that $x$ is in $L^{*}$, that each component $Y$ of $L^{*}$ is intersected by $G_{x}$. Now $G_{x}$ is contained in $L^{1}$ hence $Y$ is contained in $L^{1}$ and in turn $L^{*}$ is contained in $L^{1}$.

On the other hand, using the fact that we have a separation of $L$ and $L$ is closed there exists a neighborhood $V$ containing $L^{2}$ having the properties that the boundary $F(V)$ is disjoint with $L$ and $V$ is disjoint with $L^{1}$.

From this it follows that: $(\alpha)$ the neighborhood $V$ has at most a finite number of points in common with the compact closed set $\sum G_{x_{k}}+L^{*}$. To show this, consider any infinite set in the product. This set determines a limit point $z$ which belongs to $L^{*}$ and hence to $L^{1}$. Such a point $z$ is in $\bar{V}=V+F(V)$ and not in $F(V)$ (since $z$ is in $L$ ), hence $z$ is in $V$. This means $z$ is in $V$ and $L^{1}$ both which is impossible.

Let $p$ be any point of $L^{2}$ and consider $V$ as a neighborhood of $p$. It follows that there exists an integer $K_{1}$ such that $k>K_{1}$ implies $G_{i_{k}} \cdot V$ non-vacuous since $\left\{G_{i_{k}}\right\}$ converges to $L$. Hence there exists a component $Y_{k}$ of $G_{i_{k}}$ having the property that $Y_{k} \cdot V$ is non-vacuous for each $k>K_{1}$.

If we recall the introductory remarks concerning the relation between component-orbits and the point-orbits which they contain we see that the inequality $G_{x_{k}} \cdot \Gamma_{k} \neq 0$ holds for every component $\Gamma_{k}$ in the orbit $G_{i_{k}}$ since $x_{k} \in G_{i_{k}}$. Hence we have $G_{x_{k}} Y_{k} \neq 0$ for the particular (when $k$ is fixed) component selected in the paragraph above. This latter statement concerning $Y_{k}$ together with the fact (statement $(\alpha)$ in italics above) that $V \cdot\left(\sum G_{x k}+L^{*}\right)$ is at most finite tell us there exists an integer $K_{2}$ such that for $k>K_{2}$ the component $Y_{k}$ intersects the complement of $V$.

Combining the two properties associated with $K_{1}$ and $K_{2}$ it is clear that for $k>\max \left(K_{1}, K_{2}\right)$ each $Y_{k}$ intersects $V$ and the complement of $V$. Hence for each $k$ there exists a point $y_{k} \in Y_{k}$ such that $y_{k} \in F(V)$. This leads immediately to the conclusion that there exists a point $y$ common to $L$ and $F(V)$. By the choice of $V$ this cannot be true and the proof is complete. With the aid of Lemma $1^{*}$ we now prove the new theorem which yields the same conclusion as Lemma 2 .

Theorem. If $M$ is a compact metric space, $T(M)=M$ a pointwise periodic transformation and $\left\{G_{i}\right\}$ a convergent sequence of closed component-orbits under $T$ with limit set $L$, then $L$ is likewise a closed component-orbit.

Proof. Case (i): There exists a component $X$ of $L$ such that $X$ is isolated in $L$. 
In this case $L=G+(L-G)$, where $G$ is the component orbit of $X$ under $T$, is either a separation of $L$ or the left member is empty. This is true because $G$ is both open and closed in $L$. In view of Lemma $1^{*}$ the left side is empty; hence $L=G$ as stated by the theorem.

Case (ii): No component of $L$ is isolated in $L$.

Since $L$ is not connected let $L=L(1)+L(2)$ be some separation of $L$. By the reasoning applied in Case (i) there exists no isolated component in either $L(1)$ or $L(2)$. Now let $L(1)=L(1,1)+L(1,2)$ and $L(2)=L(2,1)+L(2,2)$ be separations of these sets and express $L$ as the sum of the four sets with double indices. Proceeding this way the proof is the same as that in Case (ii) of Lemma 2.

REMARKS. Let $\left\{G_{x_{k}}\right\}$ be a sequence of point-orbits such that the number of points in $G_{x_{k}}$ is bounded independent of $k$. It is a well known fact that if $\left\{G_{x_{k}}\right\}$ converges to a limit set $L$ then $L$ is also a point-orbit having the same bound. The theorem of this note and the simple result just mentioned are obviously of the same type in that each characterizes the limit set by the exact property it assumes for each element of a given sequence. This analogy is supported by the fact that if there is a common bound for the number of components in the orbits of the sequence the conclusion of the theorem can be augmented to include this bound for the component number of the limit set. On the other hand any improvement in the conclusion of Lemma 2 which might be suggested by this esthetic point of view (because the elements of the sequence have a minimal character) seems doomed to failure. These four possibilities form a complete set of the bounded, non-bounded, minimal and non-minimal types of sequence.

These remarks suggest that we examine certain theorems looking toward a possible analogy with periodic homeomorphisms-that is, the type of homeomorphism for which there exists a positive integer $N$ such that every point-orbit contains at most $N$ points. In this connection let us suppose that $M$ is a compact metric space and $K$ is any subset of $M$ such that $\bar{K}=M$. If $T(K)=K$ is periodic on $K$ and $S(M)=M$ is any homeomorphic extension of $T$, then $S$ is periodic on $M$. This conclusion follows by an elementary demonstration. Homeomorphic extension is used here as meaning $S(M)=M$ is a homeomorphism such that $S(x)=T(x)$ for $x \in K$. The author feels that it is not only natural to ask about such extensions for the pointwise periodic type of homeomorphism, but feels called upon to apologize for the relatively minor position the question must occupy in a note such as this. The example given below showing that the most obvious analogue of the extension theorem above does not hold came up in a 
discussion group and it was decided on an intuitive basis that the extension would be pointwise periodic. Thus one may see in this example something of the inspiration for the theory of this note, a concrete expression of that theory, and at the same time a refutation of the intuitive decision.

EXAMPLE. Let $a_{1} b_{1}$ denote an arc with end points $a_{1}$ and $b_{1}$. Similarly let $a_{11} b_{11}$ and $a_{12} b_{12}$ be arcs with end points $a_{11}, b_{11}$ and $a_{12}, b_{12}$ respectively. If we identify the 3 points $b_{1}=a_{11}=a_{12}$ and identify no other points, we obtain a triod; that is, a Y shaped figure in which three otherwise distinct arcs have a common end point. The end points of this triod are $a_{1}, b_{11}$ and $b_{12}$. Choose four more arcs calling them $a_{11 i} b_{11 i}$ and $a_{12 i} b_{12 i}$ where $i=1,2$. Identify the end points $b_{11}=a_{111}=a_{121}$ and also the end points $b_{12}=a_{121}=a_{122}$ making however no other identification. This process can be continued indefinitely, the general rule being to identify the $a$-end points of exactly two new arcs with a particular $b$-end point of the dendrite obtained in the previous stage. (Note that $a_{1}$ remains as an end point at all times.) The completed figure is to be a dendrite such that all end points other than $a_{1}$ lie on the Cantor discontinuum (middle third set) of some linear interval. The discontinuum is chosen in advance, the arcs added at each stage all have the same diameter, say $\delta_{n}$, where $\delta_{n}$ approaches zero as $n$ increases, and the arcs as added "reach" toward, but never intersect the discontinuum. This is a well known construction usually used to illustrate a dendrite with uncountably many end points.

We now denote the complete dendrite by $D$, the Cantor set of end points by $C$, and let $K=D-C$. A homeomorphism $T(K)=K$ is defined as follows: $T$ leaves each point of the arc $a_{1} b_{1}$ fixed. The arc $a_{11} b_{11}$ is sent into the arc $a_{12} b_{12}$ so that $T\left(b_{11}\right)=b_{12}$ and $T\left(b_{12}\right)=b_{11}$ and in general $p(T, x)=2$ for $x \in a_{11} b_{11}-a_{11}$. Note that $a_{11}=a_{12}=b_{1}$ is fixed. We will call this the second stage. The four $\operatorname{arcs} a_{1 j k} b_{1 j k}$ for $j=1,2$ and $k=1,2$ are permuted by $T$ so that $p .(T, x)=4$ for $x \in a_{1 j k} b_{1 j k}-a_{1 j k}$ while $T\left(b_{11}\right)=b_{12}=T\left(a_{111}\right)=a_{121}=a_{122}$ has period 2 as was defined previously. This is the third stage. In general the arcs added at the $n$th stage suffer a permutation among themselves of the period $2^{n-1}$ and the transformation agrees with the part described for the $(n-1)$ th stage in the natural way indicated by the common points.

It is clearly true that $T(K)=K$ so defined and the associated inverse $T^{-1}(K)=K$ homeomorphism are each uniformly continuous on $K$. If necessary $D$ can be constructed in the unit square using straight line segments. The high degree of symmetry allows $T$ to act as a "rigid motion" from which the uniform continuity is apparent. It follows that $T(K)=K$ may be extended to a homeomorphism $S(D)=D$ 
defined over all of $D$. However $S$ is not pointwise periodic for

$$
G_{b_{1}}=b_{1}, \quad G_{b_{11}}=b_{11}+b_{12},
$$

and so on, is a sequence of point-orbits which may be considered as component-orbits relative to themselves. This sequence converges to $C$ and $C$ is not the orbit of one of its components; that is, not the orbit of one of its points since it contains uncountably many points. The local connectivity, symmetry, and so on, of this example indicate that any conditions on $\bar{K}=M=D$ that are sufficient to make $S$ inherit the pointwise periodic property must be highly restrictive.

The University of Virginia and CORNELl University 